\title{
Synthesis of $\alpha$-Trifluoromethylated Nitrogen
}

\author{
Heterocycles.
}

Ségolène GILLE, Aurélien FERRY, Thierry BILLARD*, Bernard R. LANGLOIS.

Laboratoire SERCOF (UMR CNRS 5622). Université Claude Bernard - Lyon 1. Bât.

Chevreul. 43 Bd du 11 Novembre 1918. 69622 Villeurbanne (France).

\section{Supporting Information}

\section{Contents :}

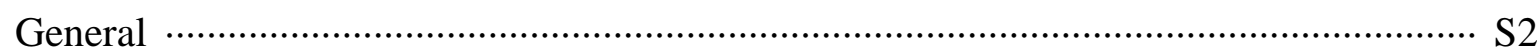

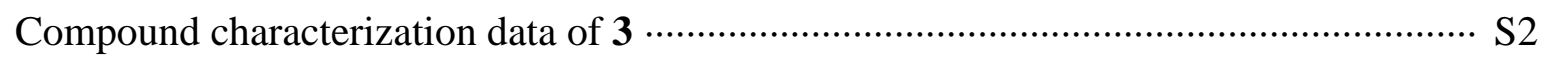

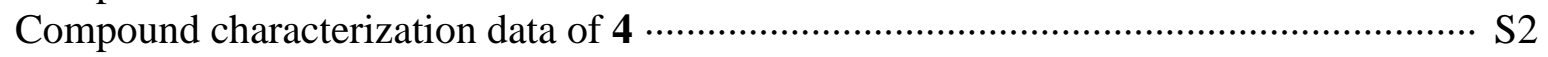

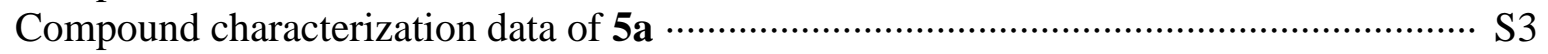

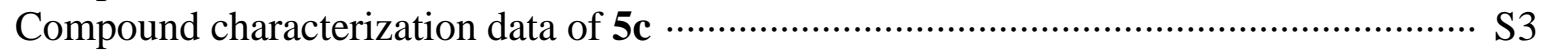

Compound characterization data of $\mathbf{5 d}$ ……………………..................................... S4

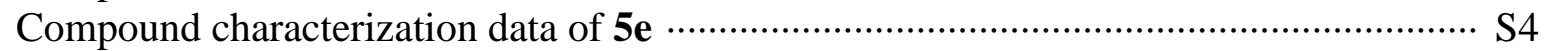

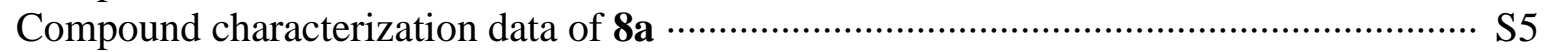

Compound characterization data of $\mathbf{8 c}$ ……………………………………………... S5

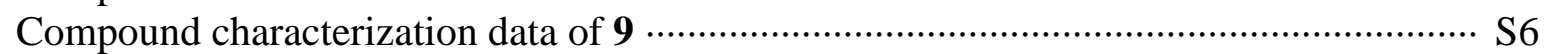

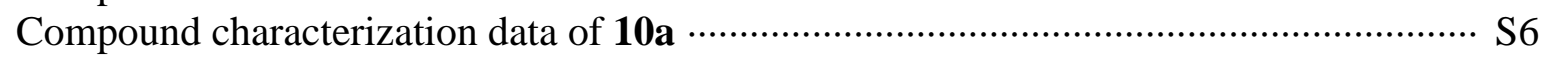

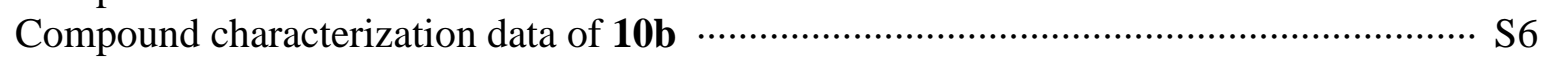

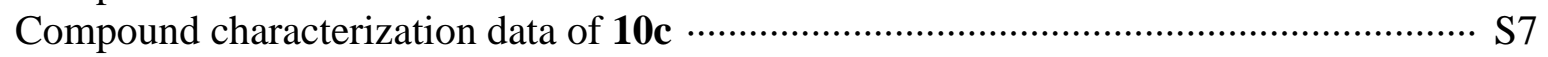

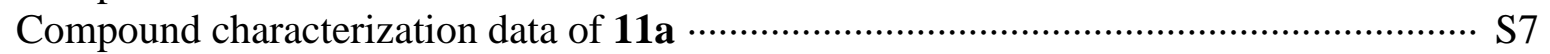

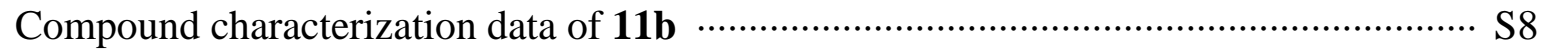

Compound characterization data of 12a ………………………………………… S8

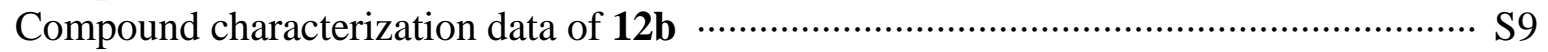

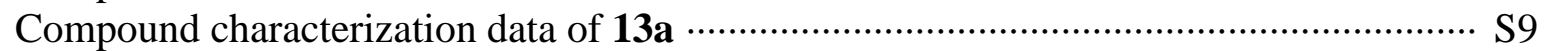

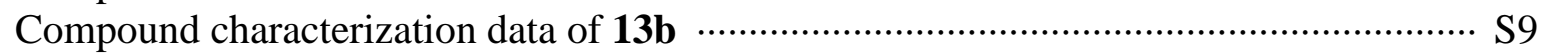

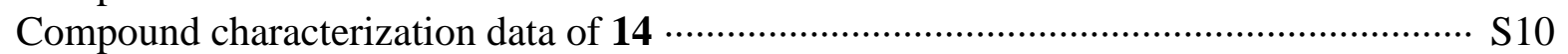

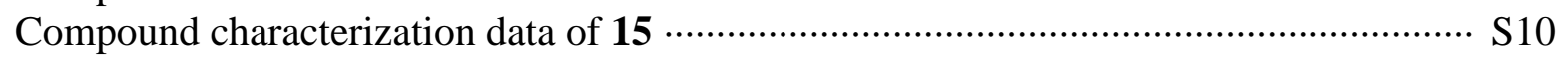

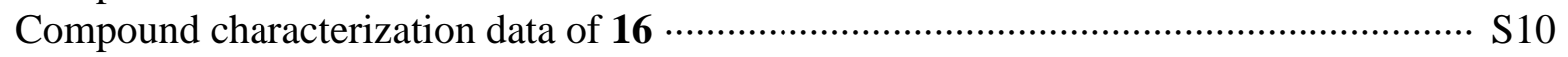

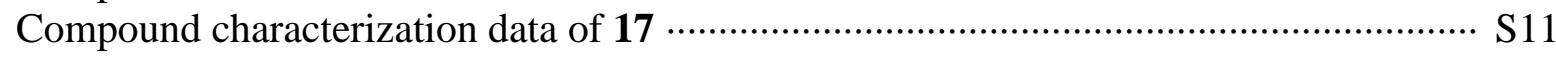

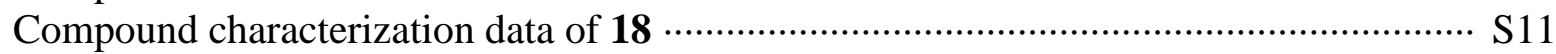

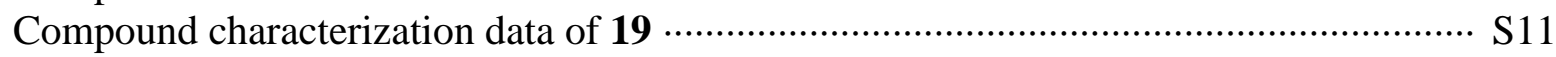


General. Solvents were distilled prior to use. Other reagents were used as received.

${ }^{1} \mathrm{H},{ }^{13} \mathrm{C}$ and ${ }^{19} \mathrm{~F}$ NMR spectra were recorded in $\mathrm{CDCl}_{3}$ at 300,75 and $282 \mathrm{MHz}$, respectively.

Chemical shifts are given in ppm relative to TMS $\left({ }^{1} \mathrm{H},{ }^{13} \mathrm{C}\right)$ or $\mathrm{CFCl}_{3}\left({ }^{19} \mathrm{~F}\right)$ as internal references. Coupling constants are given in Hertz.

Flash chromatography was performed on silica gel 60M $(0.04-0.063 \mathrm{~mm})$. Melting points (uncorrected) were determined in capillary tubes on a Büchi apparatus.

Compound $\mathbf{1}$ and $\mathbf{2}$ was prepared according to our previous work. ${ }^{10}$

\section{1,1,1-trifluoro-4-penten-2-amine hydrochloride (3) :}

$85 \mathrm{~mL}$ of a $2 \mathrm{~N}$ aqueous solution of $\mathrm{HCl}$ was dropped, at $0^{\circ} \mathrm{C}$, in a solution of $2(6.83 \mathrm{~g}$; 22.5mmol) in $\mathrm{CH}_{2} \mathrm{Cl}_{2}(30 \mathrm{~mL})$. At the end of the addition, the mixture was stirred at $50^{\circ} \mathrm{C}$ for 24h. After cooling, the crude mixture was partitioned between ether and water. Then, aqueous phase was evaporated to provide white solid (3.9g).

$\mathrm{Mp}: 210^{\circ} \mathrm{C}$ (degradation). ${ }^{1} \mathrm{H}$ NMR $\left(\mathrm{D}_{2} \mathrm{O}\right): \delta=5.64(\mathrm{ddt}, 1 \mathrm{H}, J=16.7, J=8.4, J=8.0), 5.15$ - 5.24 (massif, 2H), 4.64 (broad s, 3H), 4.04 (ddq, $1 \mathrm{H}, J=9.0, J=5.0, J=7.0), 2.58-2.68$ (massif, 1H), $2.35-2.47$ (massif, $1 \mathrm{H}) .{ }^{13} \mathrm{C}$ NMR $\left(\mathrm{D}_{2} \mathrm{O}\right): \delta=129.6,123.9(\mathrm{q}, J=280.5)$, 122.4, $51.9(\mathrm{q}, J=31.3), 30.9(\mathrm{q}, J=1.8) .{ }^{19} \mathrm{~F} \mathrm{NMR}\left(\mathrm{D}_{2} \mathrm{O}\right): \delta=-74.41(\mathrm{~d}, J=7.0)$.

Benzyl N-[1-(trifluoromethyl)-3-butenyl] carbamate (4) :

To a solution of $3(3.75 \mathrm{~g} ; 21.4 \mathrm{mmol})$ in $90 \mathrm{~mL}$ of water were added $\mathrm{NaHCO}_{3}(4.49 \mathrm{~g} ; 53.4$ mmol) and benzyl chloroformate $(3.8 \mathrm{~mL} ; 25.6 \mathrm{mmol})$. After stirring overnight, organic phase was washed with brine, dried over $\mathrm{MgSO}_{4}$ and evaporated in vacuo. A white solid was obtained after recristallisation in pentane $/ \mathrm{CH}_{2} \mathrm{Cl}_{2}$. 
(2 rotamers $90 / 10) . \mathrm{Mp}: 77-78^{\circ} \mathrm{C} .{ }^{1} \mathrm{H}$ NMR $: \delta=7.36-7.39$ (massif, $5 \mathrm{H}$ ), 5.76 (ddt, $1 \mathrm{H}, J=$ $17.1, J=9.7, J=7.3), 5.15-5.24$ (massif, $2 \mathrm{H}), 5.16(\mathrm{~s}, 2 \mathrm{H}), 4.94(\mathrm{~d}, 1 \mathrm{H}, J=8.3), 4.42$ (broad $\mathrm{m}, 1 \mathrm{H}), 2.55-2.60$ (massif, $1 \mathrm{H}$ ), $2.27-2.38$ (massif, $1 \mathrm{H}$ ). ${ }^{13} \mathrm{C} \mathrm{NMR}: \delta=156.5,136.4,131.8$, $129.0,128.7,128.5,125.5(\mathrm{q}, J=281.4), 119.9,67.8,52.7(\mathrm{q}, J=30.4), 33.2(\mathrm{q}, J=1.7) .{ }^{19} \mathrm{~F}$ NMR : $\delta=-76.48(\mathrm{~d}, J=6.9),-76.80\left(\right.$ broad s). $\mathrm{C}_{13} \mathrm{H}_{14} \mathrm{~F}_{3} \mathrm{NO}_{2}:$ calcd : $\mathrm{C}(57.14), \mathrm{H}(5.16), \mathrm{N}$ (5.13). Found : C (57.38), H (5.30), N (5.48).

Benzyl $N$-allyl- $N$-[1-(trifluoromethyl)-3-butenyl] carbamate (5a) :

To a solution of $4(273 \mathrm{mg} ; 1 \mathrm{mmol})$ in dry DMF $(1 \mathrm{~mL})$, cooled to $0^{\circ} \mathrm{C}$, was added $\mathrm{NaH}$ (60\% in mineral oil ; $48 \mathrm{mg} ; 1.2 \mathrm{mmol})$. After $10 \mathrm{~min}$, allyl bromide (1.2 mL) was added. The mixture was stirred at $\mathrm{rt}$ for $5 \mathrm{~h}$ and then hydrolysis with $\mathrm{H}_{2} \mathrm{O}(1 \mathrm{~mL})$. The crude mixture was extracted with pentane and brine. The organic phase was dried over $\mathrm{MgSO}_{4}$ and evaporated in vacuo. The crude product was purified by chromatography over silica gel.

Colorless oil (2 rotamers). ${ }^{1} \mathrm{H}$ NMR : $\delta=7.34-7.40$ (massif, 5H), $5.60-5.95$ (massif, 2H), $5.08-5.30$ (massif, 4H), 5.21(s, 2H), 5.00( broad q, 1H, $J=8.0), 3.90($ broad m, 2H), 2.57 (broad m, 2H). ${ }^{13} \mathrm{C}$ NMR : $\delta=157.4,156.1,136.5,134.6,134.2,132.4,132.2,129.0,128.9$, $128.6,128.5,128.4,128.2,125.6(\mathrm{q}, J=283.7), 125.4(\mathrm{q}, J=283.3), 119.3,119.5,117.5$, $117.3,68.30,68.35,56.7(\mathrm{q}, J=30.6), 46.3,30.4,30.5 .{ }^{19} \mathrm{~F}$ NMR $: \delta=-72.84(\mathrm{~d}, J=8.0)$. $\mathrm{C}_{16} \mathrm{H}_{18} \mathrm{~F}_{3} \mathrm{NO}_{2}$ : calcd : C (61.33), H (5.79), N (4.47). Found : C (61.51), H (5.75), N (4.18).

Benzyl $N$-(4-pentenyl)- $N$-[1-(trifluoromethyl)-3-butenyl] carbamate (5c) :

Same process than $\mathbf{5 a}$.

Colorless oil (2 rotamers). ${ }^{1} \mathrm{H}$ NMR : $\delta=7.28-7.40$ (massif, $5 \mathrm{H}$ ), $5.60-5.95$ (massif, $2 \mathrm{H}$ ), $4.60-5.30$ (massif, 7H), $3.20(\mathrm{~m}, 2 \mathrm{H}), 2.55(\mathrm{~m}, 2 \mathrm{H}), 2.05(\mathrm{~m}, 2 \mathrm{H}), 1.76(\mathrm{~m}, 2 \mathrm{H}) .{ }^{13} \mathrm{C} \mathrm{NMR}$ : $\delta=157.5,156.1,138.1,137.9,136.7,132.4,132.2,128.9,128.5,128.3,128.2,125.7(\mathrm{q}, J=$ 
283.4), $125.5(\mathrm{q}, J=283.8), 119.4,119.2,115.6,115.5,68.2,68.1,56.7(b r o a d ~ q, ~ J=30.2)$, 43.4, 31.6, 30.46, 30.38, 28.55, 28.51. ${ }^{19} \mathrm{~F}$ NMR : $\delta=-73.01(\mathrm{~d}, J=6.9),-73.15$ (broad s). $\mathrm{C}_{18} \mathrm{H}_{22} \mathrm{~F}_{3} \mathrm{NO}_{2}$ : calcd : C (63.33), H (6.50), N (4.10). Found : C (62.95), H (6.61), N (4.17).

Benzyl $N$-(5-hexenyl)- $N$-[1-(trifluoromethyl)-3-butenyl] carbamate (5d) :

Same process than $\mathbf{5 a}$.

Colorless oil (2 rotamers). ${ }^{1} \mathrm{H}$ NMR : $\delta=7.28-7.42$ (massif, 5H), $5.58-5.91$ (massif, $2 \mathrm{H}$ ), 4.65 - 5.30 (massif, 7H), $3.20(\mathrm{~m}, 2 \mathrm{H}), 2.55(\mathrm{~m}, 2 \mathrm{H}), 2.08(\mathrm{~m}, 2 \mathrm{H}), 1.65(\mathrm{~m}, 2 \mathrm{H}), 1.38(\mathrm{~m}$, $2 \mathrm{H}) .{ }^{13} \mathrm{C}$ NMR $: \delta=157.6,156.1,138.9,138.7,136.7,132.4,132.2,128.9,128.5,128.3$, $128.2,125.7(\mathrm{q}, J=283.7), 125.5(\mathrm{q}, J=284.3), 119.4,119.1,115.1,115.0,68.2,68.0,56.6$ (broad q), 43.8, 33.7, 33.6, 30.5, 30.4, 29.0, 28.1, 26.77, 26.71. ${ }^{19} \mathrm{~F}$ NMR : $\delta=-73.05$ (broad s), -73.16 (broad s). $\mathrm{C}_{19} \mathrm{H}_{24} \mathrm{~F}_{3} \mathrm{NO}_{2}$ : calcd : C (64.21), H (6.81), N (3.94). Found : C (64.38), H (6.66), N (3.72).

Benzyl $N$-(7-octenyl)- $N$-[1-(trifluoromethyl)-3-butenyl] carbamate (5e) :

Same process than $\mathbf{5 a}$.

Colorless oil (2 rotamers). ${ }^{1} \mathrm{H}$ NMR : $\delta=7.30-7.38$ (massif, $5 \mathrm{H}$ ), $5.58-5.93$ (massif, $2 \mathrm{H}$ ), 4.60 - 5.28 (massif, 7H), 3.05 - 3.31 (massif, $2 \mathrm{H}), 2.56(\mathrm{~m}, 2 \mathrm{H}), 2.04(\mathrm{~m}, 2 \mathrm{H}), 1.63(\mathrm{~m}, 2 \mathrm{H})$, $1.28-1.48$ (massif, 6H). ${ }^{13} \mathrm{C}$ NMR $: \delta=157.6,156.1,139.4,139.3,136.7,132.4,132.3$, $128.9,128.53,128.50,128.3,128.2,125.7(\mathrm{q}, J=283.2), 125.5(\mathrm{q}, J=283.4), 119.3,119.1$, 114.71, 114.67, 68.1, 68.0, 56.5 (broad m), 43.97 (broad m), 34.08, 34.05, 30.5, 30.4, 29.5, 29.2, 29.1, 29.0, 28.5, 27.34, 27.30. ${ }^{19} \mathrm{~F}$ NMR : $\delta=-72.99(\mathrm{~d}, J=6.9),-73.12$ (broad s). $\mathrm{C}_{21} \mathrm{H}_{28} \mathrm{~F}_{3} \mathrm{NO}_{2}$ : calcd : C (65.78), H (7.36), N (3.65). Found : C (65.65), H (7.47), N (3.58). 
$\underline{N \text {-(benzyloxycarbonyl)-6-(trifluoromethyl)-1,2,5,6-tetrahydropyridine (8a) : }}$

To a solution of $\mathbf{5 a}(313 \mathrm{mg} ; 1 \mathrm{mmol})$ in $\mathrm{CH}_{2} \mathrm{Cl}_{2}(10 \mathrm{~mL})$ was added $\mathbf{6}(8 \mathrm{mg} ; 0,01 \mathrm{mmol})$.

The reaction was stirred at $\mathrm{rt}$ for $5 \mathrm{~h}$. The solvent was evaporated and the crude mixture purified by chromatography over silica gel.

Yellow oil (2 rotamers 60/40). ${ }^{1} \mathrm{H}$ NMR $: \delta=7.35-7.42$ (massif, 5H), $5.65-5.80$ (massif, $2 \mathrm{H}), 5.17-5.29(\mathrm{~m}, 2 \mathrm{H}), 5.08(\mathrm{qd}, 0.6 \mathrm{H}, J=8.4, J=8.38), 4.91(\mathrm{qd}, 0.4 \mathrm{H}, J=8.4, J=8.38)$, $4.36(\mathrm{~m}, 1 \mathrm{H}), 3.75(\mathrm{~m}, 1 \mathrm{H}), 2.30-2.70$ (massif, $2 \mathrm{H}) .{ }^{13} \mathrm{C} \mathrm{NMR}: \delta=156.4,155.5,136.52$, $136.46,123.0,128.7,128.4,126.2(\mathrm{q}, J=285.6), 126.0(\mathrm{q}, J=285.6), 123.5,123.1,121.2$, $120.7,68.34,68.31,49.9(\mathrm{q}, J=30.5), 49.2(\mathrm{q}, J=30.5), 41.6,23.1,22.9 .{ }^{19} \mathrm{~F}$ NMR $: \delta=-$ $73.95(\mathrm{~d}, 0.6 \mathrm{~F}, J=8.0),-74.07(\mathrm{~d}, 0.4 \mathrm{~F}, J=9.2) . \mathrm{C}_{14} \mathrm{H}_{14} \mathrm{~F}_{3} \mathrm{NO}_{2}:$ calcd : C (58.95), $\mathrm{H}(4.95), \mathrm{N}$ (4.91). Found : C (59.02), H (5.09), N (4.82).

$\underline{N \text {-(benzyloxycarbonyl)-8-(trifluoromethyl)-1,2,3,4,7,8-hexahydroazocine (8c) }}$ :

Same process than $\mathbf{8 a}$.

Colorless oil (2 rotamers 60/40). ${ }^{1} \mathrm{H}$ NMR $: \delta=7.29-7.43$ (massif, $\left.5 \mathrm{H}\right), 5.97(\mathrm{~m}, 1 \mathrm{H}), 5.63$ $(\mathrm{m}, 1 \mathrm{H}), 5.18(\mathrm{~m}, 2 \mathrm{H}), 4.84(\mathrm{qdd}, 0.6 \mathrm{H}, J=8.2, J=12.3, J=4.1), 4.63(\mathrm{qdd}, 0.4 \mathrm{H}, J=8.1, J$ $=11.9, J=4.0), 3.91(\mathrm{~m}, 1 \mathrm{H}), 3.12(\mathrm{~m}, 1 \mathrm{H}), 2.60(\mathrm{~m}, 1 \mathrm{H}), 2.39(\mathrm{~m}, 1 \mathrm{H}), 2.17(\mathrm{~m}, 2 \mathrm{H}), 1.75$ $(\mathrm{m}, 2 \mathrm{H}) .{ }^{13} \mathrm{C} \mathrm{NMR}: \delta=157.5,156.1,136.8,136.7,135.7,135.0,128.9,128.9,128.5,128.4$, $128.1,128.0,126.0(\mathrm{q}, J=284.3), 125.8(\mathrm{q}, J=284.5), 125.2,124.8,68.1,67.9,57.3(\mathrm{q}, J=$ 29.1), $56.9(\mathrm{q}, J=29.3), 45.7,45.0,29.5,28.3,26.5,26.3,24.8,24.4 .{ }^{19} \mathrm{~F}$ NMR $: \delta=-73.50(\mathrm{~d}$, 0.6F, $J=6.9),-73.70(\mathrm{~d}, 0.4 \mathrm{~F}, J=8.0) . \mathrm{C}_{16} \mathrm{H}_{18} \mathrm{~F}_{3} \mathrm{NO}_{2}$ : calcd : C (61.33), $\mathrm{H}(5.79), \mathrm{N}(4.47)$. Found : C (61.45), H (6.04), N (4.79). 
$\underline{\text { 2-(trifluoromethyl)piperidine hydrochloride }(\mathbf{9})}$ :

To a solution of $\mathbf{8 a}(285 \mathrm{mg} ; 1 \mathrm{mmol})$ in $11 \mathrm{~mL}$ of $\mathrm{EtOH}, \mathrm{Pd} / \mathrm{C}(10 \% \mathrm{~mol} ; 0.11 \mathrm{~g})$ was added.

Then $\mathrm{H}_{2}$ was bubbled and the reaction was kept under $\mathrm{H}_{2}$ Pressure (1 atm) for $24 \mathrm{~h}$. After filtration over celite, $2 \mathrm{~mL}$ of $\mathrm{HCl}(12 \mathrm{~N})$ were dropped at $0^{\circ} \mathrm{C}$ and the mixture was stirred for 6h at rt. After extraction with $\mathrm{Et}_{2} \mathrm{O}$ and $\mathrm{H}_{2} \mathrm{O}$, aqueous phase was evaporated.

White solid. $\mathrm{Mp}=220^{\circ} \mathrm{C}($ degradation $){ }^{1} \mathrm{H}$ NMR $\left(\mathrm{D}_{2} \mathrm{O}\right): \delta=4,02(\mathrm{~m}, 1 \mathrm{H}) ; 3,50(\mathrm{~m}, 1 \mathrm{H})$; $3,07(\mathrm{~m}, 1 \mathrm{H}) ; 2,15(\mathrm{~m}, 1 \mathrm{H}) ; 1,8-2,0$ (massif, $2 \mathrm{H}) ; 1,45-1,80$ (massif, $3 \mathrm{H}$ ). ${ }^{13} \mathrm{C}$ NMR $\left(\mathrm{D}_{2} \mathrm{O}\right): \delta=123,5(\mathrm{q}, J=280,5) ; 56,8(\mathrm{q}, J=32) ; 45,7 ; 21,9(\mathrm{q}, J=1,9) ; 21,6 ; 20,6 .{ }^{19} \mathrm{~F}$ $\operatorname{NMR}\left(\mathrm{D}_{2} \mathrm{O}\right): \delta=-75,84(\mathrm{~d}, J=5,7) . \mathrm{C}_{6} \mathrm{H}_{11} \mathrm{ClF}_{3} \mathrm{~N}$ : calcd : $\mathrm{C}(38.01), \mathrm{H}(5.85), \mathrm{Cl}(18.70), \mathrm{N}$ (7.39). Found : C (37.72), H (6.07), Cl (18.52), N (7.27).

Benzyl $N$-(2-propynyl)- $N$-[1-(trifluoromethyl)-3-butenyl] carbamate (10a) :

Same process than $\mathbf{5 a}$.

Yellow oil (2 rotamers 60/40). ${ }^{1} \mathrm{H}$ NMR : $\delta=7.30-7.50$ (massif, $\left.5 \mathrm{H}\right), 5.78(\mathrm{~m}, 1 \mathrm{H}), 5.10-$ 5.30 (massif, 4H), 4.97 (qt, 0.6H, $J=8.0, J=7.7), 4.73$ (broad m, 0.4H), 3.98 - 4.22 (massif, 2H), $2.61(\mathrm{~m}, 2 \mathrm{H}), 2.56(\mathrm{~m}, 1 \mathrm{H}) .{ }^{13} \mathrm{C} \mathrm{NMR}: \delta=156.5,155.7,136.4,136.2,132.15,132.10$, $129.0,128.9,128.7,128.0,128.51,128.46,125.4(\mathrm{q}, J=284.1), 125.2(\mathrm{q}, J=283.2), 119.5$, $119.4,79.7,79.4,72.0,68.7,68.6,57.3(\mathrm{q}, J=29.8), 56.6(\mathrm{q}, J=29.6), 33.6,33.2,30.5,30.3$. ${ }^{19} \mathrm{~F}$ NMR $: \delta=-72.95(\mathrm{~d}, J=8.0) . \mathrm{C}_{16} \mathrm{H}_{16} \mathrm{~F}_{3} \mathrm{NO}_{2}$ : calcd : $\mathrm{C}(61.73), \mathrm{H}(5.18), \mathrm{N}(4.50)$. Found : C (61.79), H (5.37), N (4.31).

Benzyl $N$-(2-butynyl)- $N$-[1-(trifluoromethyl)-3-butenyl] carbamate (10b) :

Same process than $\mathbf{5 a}$.

Yellow oil (2 rotamers 60/40). ${ }^{1} \mathrm{H}$ NMR : $\delta=7.28-7.46$ (massif, $\left.5 \mathrm{H}\right), 5.80(\mathrm{~m}, 1 \mathrm{H}), 5.05-$ 5.30 (massif, 4H), 4.93 (broad m, 0.6H), 4.72 (broad s, 0.4H), $4.06(\mathrm{~m}, 2 \mathrm{H}), 2.61(\mathrm{~m}, 2 \mathrm{H})$, 
1.79 (broad m, 3H). ${ }^{13} \mathrm{C}$ NMR : $\delta=156.7,155.8,136.7,136.3,132.5,128.9,128.8,128.6$, $127.4,128.43,128.36,125.5(\mathrm{q}, J=283.5), 125.3(\mathrm{q}, J=285.6), 119.2,119.0,79.7,79.6$, $75.2,74.8,68.5,68.3,57.4($ broad q,$J=29.5), 56.8(\mathrm{q}, J=29.7), 33.6,30.5,30.3,3.9,3.7$. ${ }^{19} \mathrm{~F}$ NMR $: \delta=-72.83\left(\right.$ broad s), $-72.89(\mathrm{~d}, J=8.0) . \mathrm{C}_{17} \mathrm{H}_{18} \mathrm{~F}_{3} \mathrm{NO}_{2}:$ calcd : C (62.76), $\mathrm{H}(5.58)$, N (4.31). Found : C (62.88), H (5.65), N (4.26).

Benzyl $N$-(4-pentynyl)- $N$-[1-(trifluoromethyl)-3-butenyl] carbamate (10c) :

Same process than 5a but 1 eq. of tetrabutylammonium iodide was added before chloropentyne.

Yellow oil (2 rotamers). ${ }^{1} \mathrm{H}$ NMR $: \delta=7.30-7.42$ (massif, $\left.5 \mathrm{H}\right), 5.69(\mathrm{~m}, 1 \mathrm{H}), 4.65-5.30$ (massif, 5H), $3.31(\mathrm{~m}, 2 \mathrm{H}), 2.58(\mathrm{~m}, 2 \mathrm{H}), 2.18(\mathrm{~m}, 2 \mathrm{H}), 1.78-2.05$ (massif, $3 \mathrm{H}) .{ }^{13} \mathrm{C}$ NMR : $\delta=157.5,156.2,136.6,132.3,132.2,128.9,128.6,128.5,128.3,128.2,125.7(\mathrm{q}, J=283.2)$, $125.4(\mathrm{q}, J=283.2), 119.5,119.3,83.6,83.3,68.3,68.2,56.7($ broad q $J=30.2), 43.0,30.3$, 28.0, 16.5. ${ }^{19} \mathrm{~F}$ NMR $: \delta=-73.01(\mathrm{~d}, J=8), \delta=-73.18\left(\right.$ broad s). $\mathrm{C}_{18} \mathrm{H}_{20} \mathrm{~F}_{3} \mathrm{NO}_{2}:$ calcd $: \mathrm{C}$ (63.71), H (5.94), N (4.13). Found : C (63.95), H (6.06), N (4.01).

$\underline{N \text {-(benzyloxycarbonyl)-2-(trifluoromethyl)-5-vinyl-1,2,3,6-tetrahydropyridine (11a) : }}$

To a solution of $\mathbf{1 0 a}(311 \mathrm{mg} ; 1 \mathrm{mmol})$ in $\mathrm{CH}_{2} \mathrm{Cl}_{2}(10 \mathrm{~mL})$ was added $\mathbf{6}$ (41 $\left.\mathrm{mg} ; 0,05 \mathrm{mmol}\right)$. The reaction was stirred at $50^{\circ} \mathrm{C}$ for $24 \mathrm{~h}$. The solvent was evaporated and the crude mixture purified by chromatography over silica gel.

Colorless oil (2 rotamers 50/50). ${ }^{1} \mathrm{H}$ NMR : $\delta=7.35-7.45$ (massif, 5H), $6.32(\mathrm{~m}, 1 \mathrm{H}), 5.78$ (broad m, 1H), 4.82 - 5.30 (massif, 5H), 4.57 (m, 1H), 3.84 (m, 1H), 2.42 - 2.75 (massif, 2H). ${ }^{13} \mathrm{C}$ NMR : $\delta=156.5,155.6,140.5,140.4,136.6,136.5,136.4,136.3,129.0,128.9,128.60$, $128.56,125.75(\mathrm{q}, J=285.3), 125.80(\mathrm{q}, J=285.3), 68.4,49.1(\mathrm{q}, J=31.2), 49.8(\mathrm{q}, J=31.2)$, 
40.9, 23.1. ${ }^{19} \mathrm{~F}$ NMR $: \delta=73.99(\mathrm{~d}, 0.5 \mathrm{~F}, J=9.17),-74.13(\mathrm{~d}, 0.5 \mathrm{~F}, J=8.5) . \mathrm{C}_{16} \mathrm{H}_{16} \mathrm{~F}_{3} \mathrm{NO}_{2}:$ calcd : C (61.73), H (5.18), N (4.50). Found : C (61.62), H (4.93), N (4.23).

$\underline{N \text {-(benzyloxycarbonyl)-5-isopropenyl-2-(trifluoromethyl)-1,2,3,6-tetrahydropyridine (11b) }}$ : Same process than 11a.

Colorless oil (2 rotamers 50/50). ${ }^{1} \mathrm{H}$ NMR : $\delta=7.30-7.42$ (massif, 5H), 5.88 (broad m, 1H), $5.25(\mathrm{~m}, 2 \mathrm{H}), 4.85-5.19$ (massif, 3H), $4.65(\mathrm{~m}, 1 \mathrm{H}), 3.89(\mathrm{~m}, 1 \mathrm{H}), 2.43-2.80$ (massif, $2 \mathrm{H})$, 1.94 (broad s, 3H). ${ }^{13} \mathrm{C}$ NMR $: \delta=156.4,155.6,140.7,140.4,136.6,136.5,133.6,133.2$, $129.0,128.7,128.5,128.4,125.9(\mathrm{q}, J=286.2), 126.0(\mathrm{q}, J=286.2), 68.4,49.5(\mathrm{q}, J=30.5)$, $48.9(\mathrm{q}, J=30.5), 41.7,23.8,23.4,20.9,20.8 .{ }^{19} \mathrm{~F}$ NMR $: \delta=-73.93(\mathrm{~d}, 0.5 \mathrm{~F}, J=9.18)$, $74.07(\mathrm{~d}, 0.5 \mathrm{~F}, J=9.18) . \mathrm{C}_{17} \mathrm{H}_{18} \mathrm{~F}_{3} \mathrm{NO}_{2}$ : calcd : $\mathrm{C}(62.76), \mathrm{H}(5.58), \mathrm{N}$ (4.31). Found : $\mathrm{C}$ (62.73), H (5.75), N (4.39).

\section{$\underline{N-[1-(t r i f l u o r o m e t h y l)-3-b u t e n y l] a c r y l a m i d e ~(12 a) ~: ~}$}

To a solution of $3(878 \mathrm{mg} ; 1 \mathrm{mmol})$ in $30 \mathrm{~mL}$ of $\mathrm{CH}_{2} \mathrm{Cl}_{2}(30 \mathrm{~mL})$, cooled at $0^{\circ} \mathrm{C}, \mathrm{Et}_{3} \mathrm{~N}(1.4$ $\mathrm{mL} ; 10 \mathrm{mmol}$ ) was dropped. At the end of addition, Acryloyl chloride (543 mg; $6 \mathrm{mmol}$ ) was added, followed by DMAP $(65 \mathrm{mg} ; 0.5 \mathrm{mmol})$. The reaction was then stirred at $\mathrm{rt}$ for $24 \mathrm{~h}$. The mixture was extracted with $\mathrm{Et}_{2} \mathrm{O}$ and $\mathrm{H}_{2} \mathrm{O}$, dried over $\mathrm{MgSO}_{4}$. The solvent was evaporated.

Yellow solid. $\mathrm{Mp}=75-77^{\circ} \mathrm{C} .{ }^{1} \mathrm{H} \mathrm{NMR}: \delta=6.27(\mathrm{dd}, 1 \mathrm{H}, J=16.95, J=1.51), 6.08(\mathrm{dd}, 1 \mathrm{H}, J$ $=16.95, J=10.17), 5.99($ broad d $, 1 \mathrm{H}, J=8.29), 5.59-5.74($ massif, $2 \mathrm{H}), 5.07-5.13(\operatorname{massif}$, 2H), $4.72(\mathrm{~m}, 1 \mathrm{H}), 2.52(\mathrm{~m}, 1 \mathrm{H}), 2.30(\mathrm{~m}, 1 \mathrm{H}) .{ }^{13} \mathrm{C} \mathrm{NMR}: \delta=166.6,131.9,130.3,128.3$, $125.4(\mathrm{q}, J=281.7), 119.5,50.4(\mathrm{q}, J=30.4), 32.8 .{ }^{19} \mathrm{~F}$ NMR $: \delta=-75.97(\mathrm{~d}, J=8.03)$. $\mathrm{C}_{8} \mathrm{H}_{10} \mathrm{~F}_{3} \mathrm{NO}$ : calcd : C (49.74), H (5.22), N (7.25). Found : C (49.99), H (5.03), N (7.09). 
$\underline{N \text {-[1-(trifluoromethyl)-3-butenyl]-10-undecenamidene (12b) : }}$

Same process than $\mathbf{1 2 a}$.

Yellow oil. ${ }^{1} \mathrm{H}$ NMR : $\delta=6.37(\mathrm{~d}, 1 \mathrm{H}, J=9.6), 5.65-5.86$ (massif, $\left.2 \mathrm{H}\right), 5.15(\mathrm{~m}, 2 \mathrm{H}), 4.93(\mathrm{~m}$, 2H), $4.68(\mathrm{~m}, 1 \mathrm{H}), 2.51(\mathrm{~m}, 1 \mathrm{H}), 2.20-2.36$ (massif, 3H), $2.02(\mathrm{~m}, 2 \mathrm{H}), 1.61(\mathrm{~m}, 2 \mathrm{H}), 1.27-$ 1.36 (massif, 10H). ${ }^{13} \mathrm{C}$ NMR $: \delta=174.1,139.4,132.0,125.4(\mathrm{q}, J=281.8), 119.5,114.5$, $49.9(\mathrm{q}, J=30.2), 36.7,34.1,33.0(\mathrm{q}, J=1.8), 29.7,29.6,29.5,29.4,29.3,26.0 .{ }^{19} \mathrm{~F}$ NMR $: \delta$ $=-76.10(\mathrm{~d}, J=8.03) . \mathrm{C}_{16} \mathrm{H}_{26} \mathrm{~F}_{3} \mathrm{NO}:$ calcd $: \mathrm{C}(62.93), \mathrm{H}(8.58), \mathrm{N}(4.59)$. Found : $\mathrm{C}(62.82)$, $\mathrm{H}(8.65), \mathrm{N}(4.47)$.

6-(trifluoromethyl)-5,6-dihydro-2(1H)-pyridinone (13a) :

To a solution of 12a (193 mg; $1 \mathrm{mmol})$ in $\mathrm{CH}_{2} \mathrm{Cl}_{2}(10 \mathrm{~mL})$ was added 7 (43 $\left.\mathrm{mg} ; 0,05 \mathrm{mmol}\right)$. The reaction was stirred at $50^{\circ} \mathrm{C}$ for $24 \mathrm{~h}$. The solvent was evaporated and the crude mixture purified by chromatography over silica gel.

Colorless oil. ${ }^{1} \mathrm{H}$ NMR : $\delta=6.58(\mathrm{~m}, 1 \mathrm{H}), 5.96(\mathrm{~d}, 1 \mathrm{H}, J=10.2), 4.09(\mathrm{~m}, 1 \mathrm{H}), 2.68(\mathrm{~m}, 2 \mathrm{H})$, $1.93\left(\right.$ broad s, 1H). ${ }^{19} \mathrm{~F}$ NMR : $\delta=-78.49(\mathrm{~d}, J=6.9) . \mathrm{C}_{6} \mathrm{H}_{6} \mathrm{~F}_{3} \mathrm{NO}:$ calcd : C (43.65), H (3.66), $\mathrm{N}(8.48)$. Found : C (43.53), H (3.93), N (8.31).

\section{$\underline{\text { 14-(trifluoromethyl)aza-11-cyclotetradecen-2-one (13b) : }}$}

To a solution of $\mathbf{1 2 b}(67 \mathrm{mg} ; 0.22 \mathrm{mmol})$ in $\mathrm{CH}_{2} \mathrm{Cl}_{2}(180 \mathrm{~mL})$ was added $7(15 \mathrm{mg} ; 0,0022$ mmol). The reaction was stirred at $50^{\circ} \mathrm{C}$ for $48 \mathrm{~h}$. The solvent was evaporated and the crude mixture purified by chromatography over silica gel.

White solid. MP $=157-160^{\circ} \mathrm{C} .{ }^{1} \mathrm{H}$ NMR $: \delta=5.58(\mathrm{~d}, 1 \mathrm{H}, J=9.0), 5.45(\mathrm{dt}, 1 \mathrm{H}, J=14.6, J=$ 7.1), $5.23(\mathrm{ddd}, 1 \mathrm{H}, J=14.6, J=9.0, J=5.5), 4.64(\mathrm{~m}, 1 \mathrm{H}), 2.52(\mathrm{bm}, 1 \mathrm{H}), 2.28(\mathrm{~m}, 1 \mathrm{H})$, 1.82-2.20 (massif, 4H), 0.95-1.69 (massif, $12 \mathrm{H}) .{ }^{13} \mathrm{C}$ NMR : $\delta=173.8,135.8,125.5$ (q, $J=$ 281.9), 124.7, $50.2(\mathrm{q}, J=29.8), 37.1,31.9(\mathrm{q}, J=1.8), 31.6,26.7,26.1,25.9,25.7,24.7,24.4$. 
${ }^{19} \mathrm{~F}$ NMR $: \delta=-76.69(\mathrm{~d}, J=6.8) . \mathrm{C}_{16} \mathrm{H}_{26} \mathrm{~F}_{3} \mathrm{NO}:$ calcd : $\mathrm{C}(60.63), \mathrm{H}(8.00), \mathrm{N}(5.05)$. Found : C (60.70), H (8.27), N (4.78).

$\underline{N \text {-(diphenylmethylene) -2,2,2-chlorodifluoro-1-[(trimethylsilyl)oxy]-1-ethanamine (14) : }}$

Same process than $\mathbf{1} .^{10}$

Yellow oil. Unstable product which must be synthesized just before use. ${ }^{1} \mathrm{H}$ NMR $: \delta=7.25-$ 7.80 (massif, 10H), $5.24(\mathrm{t}, 1 \mathrm{H}, J=5.2), 0.16(\mathrm{~s}, 9 \mathrm{H}) .{ }^{13} \mathrm{C} \mathrm{NMR}: \delta=174.8,139.0,135.8$ $132.1,130.1,129.8,129.2,129.1(\mathrm{t}, J=296.7), 128.7,128.3,87.0(\mathrm{t}, J=29.4), 1.3 .{ }^{19} \mathrm{~F}$ NMR : $\delta=-68.48(\mathrm{ABX}$ system $, J=162.9, J=5.2)$.

$\underline{N \text {-(diphenylmethylene) -1,1,1-chlorodifluoro-4-penten-2-amine (15) : }}$

Same process than $2 .^{10}$

Yellow oil. ${ }^{1} \mathrm{H}$ NMR : $\delta=7.27-7.85$ (massif, $10 \mathrm{H}$ ), 5.69 (ddt, $1 \mathrm{H}, J=17.1, J=10.0, J=$ 7.2), 5.16 - 5.24 (massif, $2 \mathrm{H}), 4.15$ (ddt, $1 \mathrm{H}, J=7.6, J=5.4, J=8.3$ ), $2.74-2.80$ (massif, $2 \mathrm{H}$ ). ${ }^{13} \mathrm{C}$ NMR $: \delta=172.6,139.7,136.4,133.8,131.3,130.6(\mathrm{t}, J=295.3), 129.5,129.3,129.0$, 128.64, 128.62, 119.0, $69.1(\mathrm{t}, J=23.3), 36.1(\mathrm{dd}, J=2.5, J=1.4) .{ }^{19} \mathrm{~F}$ NMR $: \delta=-58.77$ (ABX system, $J=162.0, J=8.3) . \mathrm{C}_{18} \mathrm{H}_{16} \mathrm{ClF}_{2} \mathrm{~N}$ : calcd : C (67.61), $\mathrm{H}(5.04), \mathrm{N}$ (4.38). Found : C (67.73), H (5.31), N (4.56).

\section{1,1,1-chlorodifluoro-4-penten-2-amine hydrochloride (16) :}

Same process than $\mathbf{3}$.

White solid. $\mathrm{Mp}=200^{\circ} \mathrm{C}$ (degradation). ${ }^{1} \mathrm{H}$ NMR $\left(\mathrm{D}_{2} \mathrm{O}\right): \delta=5.71$ (massif, $\left.1 \mathrm{H}\right), 5.21-5.24$ (massif, 2H), 4.69 (broad s, 3H), 4.15 (massif, 1H), $2.71-2.80$ (massif, 1H), $2.41-2.53$ (massif, $1 \mathrm{H}) .{ }^{13} \mathrm{C}$ NMR $\left(\mathrm{D}_{2} \mathrm{O}\right): \delta=129.9,127.1(\mathrm{t}, J=293.7), 122.3,57.1(\mathrm{t}, J=27.2), 32.2$ $(\mathrm{t}, J=2.2) .{ }^{19} \mathrm{~F}$ NMR $\left(\mathrm{D}_{2} \mathrm{O}\right): \delta=-60.54(\mathrm{ABX}$ system, $J=172.9, J=7.7)$. 


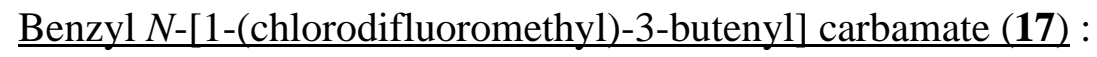

Same process than 4 .

White solid (2 rotamers 93/7). Mp $=65-66^{\circ} \mathrm{C} .{ }^{1} \mathrm{H}$ NMR $: \delta=7.30-7.40$ (massif, $5 \mathrm{H}$ ), 5.77 (ddt, $1 \mathrm{H}, J=16.7, J=9.6, J=7.2), 5.10-5.24$ (massif, $5 \mathrm{H}), 4.50(\mathrm{ddt}, 1 \mathrm{H}, J=3.5, J=18, J$ $=9.2), 2.65(\mathrm{~m}, 1 \mathrm{H}), 2.32(\mathrm{~m}, 1 \mathrm{H}) .{ }^{13} \mathrm{C} \mathrm{NMR}: \delta=156.3,136.3,132.0,129.8(\mathrm{t}, J=297.2)$, 129.0, 128.7, 128.5, 119.9, 67.9, $57.9(\mathrm{t}, J=26.4), 34.2 .{ }^{19} \mathrm{~F}$ NMR $: \delta=-61.24$ (ABX system, 0.93F, $J=164.0, J=8.6$ ), -61.39 (broad ABX system, 0.7F, $J=165.0) . \mathrm{C}_{13} \mathrm{H}_{14} \mathrm{ClF}_{2} \mathrm{NO}_{2}$ : calcd : C (53.90), H (4.87), Cl (12.24), N (4.83). Found : C (53.82), H (4.91), Cl (12.51), N (4.71).

Benzyl $N$-allyl- $N$-[1-(chlorodifluoromethyl)-3-butenyl] carbamate (18) :

Same process than $\mathbf{5 a}$.

Colorless oil (2 rotamers). ${ }^{1} \mathrm{H}$ NMR : $\delta=7.35-7.38$ (massif, 5H), $5.60-6.01$ (massif, $2 \mathrm{H}$ ), $4.90-5.30$ (massif, 7H), $3.92(\mathrm{~m}, 2 \mathrm{H}), 2.60(\mathrm{~m}, 2 \mathrm{H}) .{ }^{13} \mathrm{C} \mathrm{NMR}: \delta=157.5,156.1,136.6$, 134.9, 134.4, 132.6, 132.5, $129.9(\mathrm{t}, J=298.6), 129.7(\mathrm{t}, J=299.2), 128.92,128.86,128.6$, $128.5,128.4,128.2,119.4,119.2,117.5,117.3,68.3,68.3,62.4(\operatorname{broad} \mathrm{m}), 46.4,30.6 .{ }^{19} \mathrm{~F}$ NMR : $\delta=-58.56($ broad $\mathrm{ABX}$ system, $J=167.0) . \mathrm{C}_{16} \mathrm{H}_{18} \mathrm{ClF}_{2} \mathrm{NO}_{2}$ : calcd : $\mathrm{C}(58.27), \mathrm{H}$ (5.50), Cl (10.75). Found : C (58.49), H (5.38), Cl (11.02).

$\underline{N \text {-(benzyloxycarbonyl)-6-(chlorodifluoromethyl)-1,2,5,6-tetrahydropyridine (19) : }}$

Same process than $\mathbf{8 a}$.

Colorless oil (2 rotamers). ${ }^{1} \mathrm{H}$ NMR : $\delta=7.32-7.44$ (massif, $\left.5 \mathrm{H}\right), 5.61-5.84$ (massif, $2 \mathrm{H}$ ), $4.89-5.30$ (massif, 3H), $4.39(\mathrm{~m}, 1 \mathrm{H}), 3.74(\mathrm{~m}, 1 \mathrm{H}), 2.56(\mathrm{~m}$ large, $2 \mathrm{H}) .{ }^{13} \mathrm{C}$ NMR : $\delta=$ $156.4,155.6,136.6,136.5,130.5(\mathrm{t}, J=301.3), 130.3(\mathrm{t}, J=301.6), 129.0,128.7,128.6$, 
$128.4,123.5,123.0,121.6,121.1,68.3,41.5,41.4,23.7,23.5,55.5(\mathrm{dd}, J=26.9, J=24.1)$, $54.8(\mathrm{dd}, J=26.9, J=23.6) .{ }^{19} \mathrm{~F} \mathrm{NMR}: \delta=-59.34(\mathrm{ABX}$ system, $J=163.2, J=12.9),-59.35$ (ABX system, $J=162.3, J=12.6) . \mathrm{C}_{14} \mathrm{H}_{14} \mathrm{ClF}_{2} \mathrm{NO}_{2}:$ calcd : $\mathrm{C}$ (55.73), $\mathrm{H}$ (4.68), $\mathrm{Cl}$ (11.75), $\mathrm{N}$ (4.64). Found : C (55.86), H (4.70), Cl (11.93), N (4.55). 